\title{
Produktion in der Industrie 4.0: Renaissance der arbeitswissenschaftlichen Forschung für manuelle Montagesysteme
}

\author{
Christopher Brandl ${ }^{1}$ Alexander Mertens ${ }^{1} \cdot$ Holger Luczak ${ }^{1}$. Verena Nitsch ${ }^{1}$
}

๑) Springer-Verlag GmbH Deutschland, ein Teil von Springer Nature 2018

Manuelle Montagesysteme haben in historischer Perspektive - hier beginnend mit dem Ende des 2. Weltkriegs - immer wieder und mit verschiedenen Ansätzen die arbeitswissenschaftliche Analytik und Gestaltungsthematik herausgefordert.

In den 1950er Jahren waren es mit der Verbreitung industrieller Groß- und Mittelserienfertigung, die aus Amerika importierten Systeme vorbestimmter Zeiten (SVZ), die das arbeitswissenschaftliche Forschungs- und Praxisfeld anregten. Auf dem damals fruchtbaren Boden des REFA-Arbeitsund Zeitstudiums gediehen die SVZ-Forschungen zur Validität und Planungssicherheit von Vorab-Gestaltungsvarianten von Montage-Arbeitsplätzen. Entlang der Leitlinien der Bewegungsverdichtung und Bewegungsvereinfachung wurden so Entwürfe von Montagearbeitsplätzen realisiert, die im Wesentlichen zu kurzzyklisch repetitiven Arbeitsinhalten passten. Auch heute noch gehen in etliche CADSysteme mit Menschmodellierung sowie in rechnergestützte Planungsalgorithmen Grundgedanken und Datensätze der SVZ ein.

In den 1960er Jahren wandelte sich mit den ersten Kundenansprüchen an Variantenvielfalt der Fokus arbeitswissenschaftlicher Bemühungen in Richtung Sensomotorik und Anlernoptimierung, da das Montagepersonal nun mehrere Produktvarianten und Montagearbeitsplätze zu bewältigen hatte. Die ehemals rein technisch-ökonomisch orientierten Rationalisierungen der SVZ wurden erstmals um menschbezogene Ansätze, Argumentenbilanzen und Gestaltungshinweise ergänzt.

In den 1970er Jahren kam die Welle wissenschaftlich begründeter Kritik an den kurzzyklischen repetitiven Arbeitsinhalten in der manuellen Montage zum Tragen. Arbeitsphysiologisch und arbeitsmedizinisch tätige Arbeitswissenschaftler konnten nachweisen, dass die immer

Christopher Brandl

c.brandl@iaw.rwth-aachen.de

1 Lehrstuhl und Institut für Arbeitswissenschaft, RWTH Aachen University, Aachen, Deutschland wiederkehrende Beanspruchung einzelner Muskelgruppen und Muskelfasern längerfristige Schädigungen verursachen kann. Besonderen Einfluss gewann die Humanisierungsforschung der Arbeitspsychologen, die auf Demotivation und Absentismus aufmerksam machte und damit auch die Leistungsseite des Problems thematisierte. Ergonomisch wurden Entlastungen, z.B. in Bezug auf Körperhaltungen und Arbeitsdauern angestrebt. Organisatorisch wurden zunächst die klassischen Instrumente von Arbeitsstrukturierung (Job-Rotation, -Enlargement, -Enrichment) bemüht, die in vielfältige Formen von Gruppenarbeit - auch nach Team Performance Modellen - entwickelt wurden. Technisch wurden Montagelinien und Montagearbeitsplätze mit Werkzeugen und Vorrichtungen ausgestattet, die Spitzenbelastungen reduzierten, Kommunikation und Kooperation ermöglichten und leistungsfördernd waren.

In den 1980er Jahren rollte die „CIM-Welle“ (Computer Integrated Manufacturing), die der Zielvorstellung kompletter Automatisierung in der Produktion verpflichtet war. Die Planungshoheit für Montagesysteme verlagerte sich von der Arbeitswissenschaft weg in Richtung Produktionssystematik und Informatik, was sich u. a. daran zeigt, dass in Referenzmodellen für die industrielle Produktion einschließlich Montage (Y-Modell zum CAD/CAM, Aachner PPS-Modell) ein Menschbezug kaum erkennbar war. Konsequenz waren „Restarbeitsplätze“, deren Automatisierung sich nach technischen und ökonomischen Kriterien als schwierig oder unmöglich erwies, da z. B. Flexibilitätsanforderungen nur durch menschlichen Arbeitseinsatz zu bewältigen waren.

In den 1990er Jahren und nach dem Jahrtausendwechsel waren im Zusammenhang mit einer Informatisierung der Arbeitswissenschaft als Software-Ergonomie, MenschComputer-Interaktion und Work With Computing Systems manuelle Montagesysteme nur insofern im Fokus arbeitswissenschaftlicher Forschung und Entwicklung, als computerbasierte Arbeitsplatzkonstruktionen und -konfigurationen, Visualisierungen von Arbeitsabläufen und Planungsalgorithmen auf der Basis von digitalen Mensch-Modellen 
mit unterschiedlichen Aspekten auch als Anwendungsfälle für diese Instrumentarien thematisiert wurden.

Umso bemerkenswerter - bei genauer Betrachtung jedoch die logische Folge der rasanten Technologieentwicklung und Forschung im Bereich Industrie 4.0 - erscheint uns die aktuelle Renaissance manueller Montagesysteme in der arbeitswissenschaftlichen Forschung, Entwicklung und Praxis. Die Identifikation einer Renaissance basiert auf dem Fakt, dass unser Aufruf, zu einem ZfA-Sonderheft mit Schwerpunkt Produktion beizutragen, von der Fachlandschaft mit sechs Vorschlägen bedient wurde, die explizit die Montage zum Gegenstand haben. Keine Frage also, dass die Montage aktuell in der Arbeitswissenschaft wieder angekommen ist und deutlich prioritär an verschiedenen Forschungsstellen und Anwendungsstätten behandelt wird. Insbesondere der aktuelle Bedarf und (zumeist technische) Möglichkeiten der Unterstützung bei der Montage - aber auch bei vor- und nachgelagerten Arbeitsprozessen - stehen in diesem Sonderheft im Fokus.

$\mathrm{Zu}$ Beginn führen M. Weber und S. Stowasser in die Thematik der kollaborierenden Robotersysteme im Bereich der Produktion ein und zeigen zugleich Einsatzmöglichkeiten zur Verbesserung der Ergonomie an ausgewählten Praxisbeispielen aus der deutschen Metall- und Elektroindustrie auf.

Der Einsatz unterstützender Roboter wird auch im Beitrag von J. Hegenberg und Mitautoren beleuchtet. Auf Grundlage einer praxisnahen empirischen Evaluation eines robotergestützten Montagearbeitsplatzes werden hier mögliche Bedingungen für eine Effizienzsteigerung und Beanspruchungsreduktion in der Automobilherstellung identifiziert.

Vergleichbar praxisnah und informativ in Bezug auf den zielgerichteten Einsatz technischer Assistenzsysteme in Montage und Logistik ist auch der Forschungsbeitrag von R. Hensel und M. Keil der Audi AG. Dieser beschreibt eine Reihe von Feldstudien, anhand derer die Effektivität passiver Exoskelette, u.a. in der Beinunterstützung bei Stehtätigkeiten und der Rückenunterstützung bei Lastenmanipulationen, beurteilt wird.

Neben den verschiedenen technischen Möglichkeiten der vorrangig physischen Unterstützung, versprechen verschiedene Assistenzsysteme auch bei primär kognitiven Anforderungen eine Belastungsreduktion. M. Bornewasser und Mitautoren befassen sich entsprechend mit informatorischen Assistenzsystemen, die einer Entscheidungsun- terstützung bei komplexen Montageprozessen gewidmet sind.

Weitere technische Möglichkeiten für die Gestaltung individualisierbarer Montagearbeitsplätze beleuchten S. Schlund und Mitautoren. Ihre Betrachtungen bilden u. a. eine arbeitswissenschaftliche Grundlage für die experimentelle Evaluation unterschiedlicher Kombinationen individualisierbarer Parameter und für die Ableitung allgemeingültig anwendbarer Gestaltungsregeln.

Besonders relevant werden die Möglichkeiten einer individualisierten Arbeitsgestaltung vor dem Hintergrund des demografischen Wandels, der einer altersdifferenzierten Betrachtungsweise bedarf, die auch physische Eigenschaftskomponenten besonders berücksichtigt. Vor diesem Hintergrund beschreiben K. Börner und A. Bullinger ihre Feldstudie zur Beanspruchung von Montagearbeitern in der Motorendmontage und verdeutlichen damit die Notwendigkeit einer altersdifferenzierten Arbeitsgestaltung in diesem Tätigkeitsbereich.

Individuelle (physische) Parameter des Menschen sind bislang auch in der Arbeitsgestaltung von Kommissioniertätigkeiten in Kühllagern und Tiefkühllagern sowie entsprechenden berufsgenossenschaftlichen Regeln noch unzureichend berücksichtigt, wie S. Groos und Mitautoren in ihrem praxisorientierten Beitrag zur Untersuchung des Arbeitszeit-Pausenzeit-Regimes für körperlich schwere Arbeit in Kälte verdeutlichen.

Auch in der Beurteilung von Belastungen durch Körperhaltung gibt es weiteren Differenzierungsbedarf. So zeigen T. Hellig und Mitautoren am Beispiel einer empirischexperimentellen Laborstudie, dass Wechselwirkungen von Körperteilhaltungen des Rückens und der Schulter die Beanspruchung deutlich erhöhen und somit in der Bewertung von Körperhaltungen systematisch berücksichtigt werden sollten.

Schließlich darf auch in einem Sonderheft mit dem Fokus Produktion die stark in ihrer Bedeutung zunehmende Problematik der psychischen Belastungen nicht unberücksichtigt bleiben. K. Sonntag und E. Feldmann leisten hierzu einen wichtigen Beitrag mit einem Überblick über aktuelle psychische Belastungen in der Arbeitswelt und der Vorstellung eines praxisorientierten Verfahrens zur Gefährdungsbeurteilung Psychische Belastung (GPB).

Wir danken den Autorinnen und Autoren für ihre positive Reaktion auf unsere Initiative und freuen uns, dem ZfALeserkreis ein Sonderheft mit spannenden, zukunftsweisenden und praxisorientierten Beiträgen vorlegen zu können. 